\title{
Direct Electrochemical Synthesis of Bismuth (III) Thiolates/Dithiolates and Their Coordination Compounds
}

\author{
Harpreet Kaur ${ }^{1}$, Baljit Singh $^{2}$ \\ ${ }^{I}$ (Chemistry Department, Punjabi University, Patiala-147002, India) \\ ${ }_{2}^{2}$ (Chemistry Department, Punjabi University, Patiala-147002, India)
}

\begin{abstract}
Bismuth (III) thiolates/dithiolates have been synthesized by electrochemical reactions of ethanethiol, 2-propanethiol, 2-methylpropane-2-thiol, Butanethiol, 1-pentanethiol, , Ethane- 1, 2-dithiol and Butane-1, 4dithiol at sacrificial bismuth anode and inert platinum cathode using tetrabutylammonium chloride as supporting electrolyte. The coordination compounds of these thiolates/dithiolates with 1, 10-phenanthroline and 2, 2'-bipyridyl have also been synthesized electrochemically. The solid products that separated in the anode compartment have been isolated and characterized by elemental analysis and infrared spectral studies. Current efficiencies of these reactions are quite high.
\end{abstract}

Keywords: H-type cell, electrochemical synthesis, Bismuth (III) thiolates/dithiolates, current efficiencies, sacrificial electrode.

\section{Introduction}

Electrochemical technique is the direct route to carry out oxidation or reduction without introducing any foreign oxidizing or reducing agent [1-3]. It has been used as synthetic method in organic chemistry [4-6] for last sixteen decades. In medical field, bismuth compounds are used to relieve diaper rash, treat burns as well as to treat gastric disorders such as diarrhea [7, 8]. Importantly these compounds are effective against bacterium, Helicobacter pylori, which causes peptic ulcers $[9,10]$. In light of recent interest in the medicinal importance of bismuth compounds we are developing some simple compounds using electrochemical synthetic technique.

\section{Experimental}

Acetonitrile was kept over phosphorus pentoxide for $24 \mathrm{~h}$ and then double distilled. Freshly distilled acetonitrile was used as solvent in all these reactions. Tetrabutylammonium chloride (Reidal pure) was crystallized from conductivity water and dried under reduced pressure at $100^{\circ} \mathrm{C}$. It was then used as supporting electrolyte in all these reactions. An H-type cell made of pyrex glass in which the cathode and anode compartments were separated from each other by a sintered glass disc of G-3 porosity, was used as the reaction vessel. Both compartments were provided with two openings; one for guard tube and the other for electrode. Platinum guage $\left(2.0 \times 1.0 \times 1.0 \mathrm{~cm}^{3}\right)$ was used as cathode and bismuth rod $\left(2.0 \times 10.0 \times 0.2 \mathrm{~cm}^{3}\right)$ as anode. Direct current was obtained with the help of Toshniwal electrophoresis power supply. The electrolytic solution in the anode compartment was stirred efficiently using magnetic stirrer. $1.0 \mathrm{~mL}$ of thiol, $1.0 \mathrm{~g}$ of tetrabutylammonium chloride and $250 \mathrm{~mL}$ of freshly distilled acetonitrile were taken in the H-type cell. Bismuth metal electrode was dipped in anode compartment and platinum guage in the cathode compartment and outlets were sealed after fitting the guard tubes. Necessary connections were made with power supply and potential across the electrodes was then adjusted so that a current of $20 \mathrm{~mA}$ passed through the solution. The cell can be represented as:

$$
\mathrm{Bi}_{(+)}\left|\begin{array}{c}
\mathrm{CH}_{3} \mathrm{CN}+\mathrm{Bu}_{4} \mathrm{NCl}+ \\
\text { Thiol }
\end{array}\right| \mathrm{Pt}_{(-)}
$$

Where:

$\mathrm{Bi}_{(+)}$is Bismuth anode.

$\mathrm{Pt}_{(-)}$is Platinum cathode.

$\mathrm{Bu}_{4} \mathrm{NCl}$ is supporting electrolyte.

The electrolysis was carried out with continuous stirring in the anode compartment. After conducting electrolysis for twelve hours, the product was filtered, washed with hot acetonitrile and dry ether and finally dried under vacuum. The current efficiencies (gram equivalents of metal dissolved per faraday of electricity passed) of all these reactions were determined by electrolyzing the above systems for exactly two hours at a constant current of $20 \mathrm{~mA}$ as reported earlier [3]. Melting point of all these products was recorded using electrical device with heating rate of $5^{\circ} \mathrm{C}$ per minute. Infrared spectra of the products were recorded on PerkinElmer spectrophotometer (RXI) in the region of $4000-450 \mathrm{~cm}-1$ using potassium bromide pellets. The products 
were analyzed for bismuth contents. Weighed amount of the product was heated to dry mass with fuming nitric acid six times. The dry mass was dissolved in few drops of dilute nitric acid and $100 \mathrm{~mL}$ solution was made. The contents of bismuth in solution were estimated volumetrically by oxine method [11]. The microanalyses of $\mathrm{C}, \mathrm{H}$ and $\mathrm{S}$ of these compounds have also been carried out.

\subsection{The coordination compounds of bismuth thiolates and dithiolates}

Bismuth thiolates and dithiolates were refluxed with 1, 10-phenanthroline or 2,2'-bipyridyl in different solvents like benzene, chloroform, carbon tetrachloride, DMSO, diethyl ether etc. for more than $48 \mathrm{~h}$. However, the elemental analysis shows that these donor molecules could not rupture the thiolate bridges in these compounds to form their coordination compounds. It was therefore decided to add the ligand to these thiolates and dithiolates before these are polymerized. Therefore in addition to supporting electrolyte, $1.0 \mathrm{~g}$ of the ligand has also been added to these systems and the solution was electrolyzed for $8 \mathrm{~h}$. The solid product separate in anode compartment was filtered, washed with dry ether and dried under vacuum. The analytical data conform to $\mathrm{Bi}(\mathrm{SR}) 3 . \mathrm{L}$ in case of thiols and $\mathrm{BiS} 2(\mathrm{CH} 2)$ n.L in case of dithiols.

$$
\mathrm{Bi}_{(+)}\left|\begin{array}{c}
\mathrm{CH}_{3} \mathrm{CN}+\mathrm{Bu}_{4} \mathrm{NCl}+ \\
\text { Thiol }+\mathrm{L}
\end{array}\right| \quad \mathrm{Pt}_{(-)}
$$

Where:

$\mathrm{Bi}(+)$ is Bismuth anode.

$\mathrm{Pt}(-)$ is Platinum cathode.

$\mathrm{Bu} 4 \mathrm{NCl}$ is supporting electrolyte

$\mathrm{L}$ is 1,10-phenanthroline or 2,2'-bipyridyl

\section{Results and Discussion}

The products obtained from electrolysis of thiols in acetonitrile and tetrabutylammonium chloride are insoluble in commonly used organic solvents like chloroform, benzene, methanol, acetone, dimethyl sulphoxide, $\mathrm{N}, \mathrm{N}$-dimethyl formamide etc. All these compounds do not melt upto 300o C, however, colour of these compounds changes around $230 \mathrm{o} \mathrm{C}$ thereby indicating that these products decompose around this temperature. The analytical data (bismuth, carbon, and hydrogen contents in all these products) along with their electrolytic characteristics of these systems are summarized in Table-I.

Table-I: Electrolysis Characteristics, Analytical and other Related Data of Electrolysis of Thiols at Bismuth

\begin{tabular}{|c|c|c|c|c|c|c|c|c|c|}
\hline \multicolumn{10}{|c|}{ Anode } \\
\hline \multirow[t]{2}{*}{ System } & \multirow[t]{2}{*}{$\begin{array}{c}\text { Potenti } \\
\text { al }\end{array}$} & \multirow{2}{*}{$\begin{array}{c}\text { Electricity } \\
\text { Passed } \\
(\text { Coulomb) }\end{array}$} & \multirow[t]{2}{*}{ Product } & \multirow[t]{2}{*}{ Colour } & \multicolumn{4}{|c|}{$\begin{array}{l}\text { Elemental Analysis } \\
\text { Found/(Calc.)\% }\end{array}$} & \multirow{2}{*}{$\begin{array}{c}\text { Current Efficiencies } \\
\text { (Gram Equivalent/ } \\
\text { Faraday) }\end{array}$} \\
\hline & & & & & $\mathrm{Bi}$ & $\mathrm{C}$ & $\mathrm{H}$ & $\mathrm{S}$ & \\
\hline Ethanethiol & 40 & 864 & $\mathrm{C}_{6} \mathrm{H}_{15} \mathrm{~S}_{3} \mathrm{Bi}$ & Pink & $\begin{array}{c}50.8 \\
(53.3)\end{array}$ & $\begin{array}{c}17.9 \\
(18.3)\end{array}$ & $\begin{array}{c}1.9 \\
(3.82)\end{array}$ & $\begin{array}{c}21.3 \\
(24.4)\end{array}$ & 0.94 \\
\hline 2- propanethiol & 60 & 864 & $\mathrm{C}_{9} \mathrm{H}_{24} \mathrm{~S}_{3} \mathrm{Bi}$ & Yellow & $\begin{array}{c}46.4 \\
(48.0) \\
\end{array}$ & $\begin{array}{c}21.2 \\
(24.8)\end{array}$ & $\begin{array}{c}0.6 \\
(4.83) \\
\end{array}$ & $\begin{array}{l}21.0 \\
(22.1) \\
\end{array}$ & 0.93 \\
\hline $\begin{array}{c}\text { 2-methylpropane- } \\
\text { 2-thiol }\end{array}$ & 50 & 864 & $\begin{array}{c}\mathrm{C}_{12} \mathrm{H}_{27} \mathrm{~S}_{3} \mathrm{~B} \\
\mathrm{i}\end{array}$ & Yellow & $\begin{array}{c}43.5 \\
(43.9)\end{array}$ & $\begin{array}{c}28.7 \\
(30.2)\end{array}$ & $\begin{array}{c}1.9 \\
(5.6)\end{array}$ & $\begin{array}{c}18.7 \\
(20.1)\end{array}$ & 0.86 \\
\hline Butanethiol, & 40 & 864 & $\begin{array}{c}\mathrm{C}_{12} \mathrm{H}_{27} \mathrm{~s}_{3} \mathrm{~B} \\
\mathrm{i}\end{array}$ & Orange & $\begin{array}{c}41.0 \\
(43.9)\end{array}$ & $\begin{array}{c}31.0 \\
(30.25)\end{array}$ & $\begin{array}{c}4.3 \\
(5.6)\end{array}$ & $\begin{array}{c}18.6 \\
(20.1)\end{array}$ & 0.87 \\
\hline 1-pentanethiol & 40 & 864 & $\begin{array}{c}\mathrm{C}_{15} \mathrm{H}_{33} \mathrm{~S}_{3} \mathrm{~B} \\
\mathrm{i} \\
\end{array}$ & Pink & $\begin{array}{c}39.1 \\
(40.3) \\
\end{array}$ & $\begin{array}{c}33.3 \\
(34.7) \\
\end{array}$ & $\begin{array}{l}55.5 \\
(6.3)\end{array}$ & $\begin{array}{c}17.2 \\
(18.5) \\
\end{array}$ & 0.96 \\
\hline $\begin{array}{l}\text { Ethane- 1, 2- } \\
\text { dithiol }\end{array}$ & 50 & 864 & $\mathrm{C}_{6} \mathrm{H}_{15} \mathrm{~S}_{6} \mathrm{Bi}$ & Purple & $\begin{array}{c}40.6 \\
(42.8)\end{array}$ & $\begin{array}{c}11.3 \\
(14.7)\end{array}$ & $\begin{array}{c}0.8 \\
(3.1)\end{array}$ & $\begin{array}{c}25.6 \\
(39.3)\end{array}$ & 0.83 \\
\hline Butane-1, 4-dithiol & 50 & 864 & $\begin{array}{c}\mathrm{C}_{12} \mathrm{H}_{27} \mathrm{~S}_{6} \mathrm{~B} \\
\mathrm{i}\end{array}$ & Purple & $\begin{array}{c}34.8 \\
(36.5)\end{array}$ & $\begin{array}{c}21.6 \\
(25.1)\end{array}$ & $\begin{array}{c}0.6 \\
(4.7)\end{array}$ & $\begin{array}{c}31.3 \\
(33.5)\end{array}$ & 0.84 \\
\hline
\end{tabular}

Perusal of Table-I reveals that the analytical data conform to the molecular formula $\mathrm{Bi}(\mathrm{SR}) 3$ and BiS2 $(\mathrm{CH} 2) n$.The infrared spectral data of these products reveals that the characteristic bands appear in the region of 559-530 cm-1 and 530-470 cm-1. However the band due to $v(\mathrm{~S}-\mathrm{H})$ which generally appears around 2603-2499 cm-1 is missing in the infrared spectra of these products. Survey of literature [12] reveals that $v(\mathrm{M}-$ S) stretching bands appear in the region of 400-250 cm-1. The bands present in the region of 592-410 cm-1 are attributed to $v(\mathrm{Bi}-\mathrm{S})$ stretching vibration $[13,14]$ of bridged metal thiolate and dithiolate indicating the polymeric nature of these compounds. The bands which are appearing in the region of $692-659 \mathrm{~cm}-1$ are also in agreement with the $v(\mathrm{C}-\mathrm{S})$ stretching modes reported in literature $[15,16]$. The insoluble behavior of these 
compounds in various organic solvents also supports the polymeric nature of these compounds. Current efficiencies of some of these systems have also determined which are quite close to $100 \%$ thereby indicating that the reactions leading to the formation of these thiolates and dithiolates are the predominant reactions of these systems. The reaction scheme for the formation of products is detailed below:

At inert cathode:

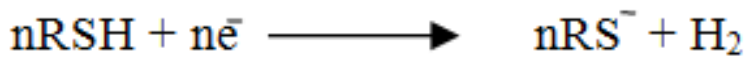

At sacrificial anode:

$$
\mathrm{nRS}^{-}+\mathrm{M}_{(+)} \longrightarrow \mathrm{M}(\mathrm{SR})_{\mathrm{n}}+\mathrm{ne}^{-}
$$

Survey of literature reveals [17] that $v(\mathrm{C}-\mathrm{N})$ in 1,10-phenanthroline or 2,2'-bipyridyl appear around 1310-1360 cm-1 . It has been observed that in these complexes these bands appear at 1600-1585 cm-1 higher than 1,10-phenanthroline or 2,2'-bipyridyl. Infrared spectra of these compounds also exhibit peaks in the region of $695-657 \mathrm{~cm}-1,578-558 \mathrm{~cm}-1$ and $472-428 \mathrm{~cm}-1$ [18]. These bands are assigned to $v(\mathrm{C}-\mathrm{S}), v(\mathrm{Bi}-\mathrm{S})$ terminal and $v(\mathrm{Bi}-\mathrm{S})$ bridged stretching vibrations respectively. Shift of these bands indicates that the ligand has been added to these systems. These coordinate compounds are also insoluble in commonly used organic solvents like benzene, methanol, acetone, dimethyl sulphoxide, $\mathrm{N}, \mathrm{N}$-dimethyl formamide etc thereby indicating that these could be polymeric in nature like their parent bismuth thiolate/dithiolate.

Table-II: Electrolysis Characteristics, Analytical and other Related Data of Electrolytic product of various thiol Systems + 1, 10-phenanthroline at Bismuth Anode

\begin{tabular}{|c|c|c|c|c|c|c|c|c|c|}
\hline \multirow[t]{2}{*}{ System } & \multirow[t]{2}{*}{$\begin{array}{c}\text { Potenti } \\
\text { al }\end{array}$} & \multirow{2}{*}{$\begin{array}{l}\text { Electricity } \\
\text { Passed } \\
\text { (Coulomb) }\end{array}$} & \multirow[t]{2}{*}{ Product } & \multirow[t]{2}{*}{ Colour } & \multicolumn{4}{|c|}{$\begin{array}{l}\text { Elemental Analysis } \\
\text { Found/(Calc.) } \%\end{array}$} & \multirow{2}{*}{$\begin{array}{c}\text { Current } \\
\text { Efficiencies } \\
\text { (Gram Equivalent/ } \\
\text { Faraday) }\end{array}$} \\
\hline & & & & & $\mathrm{Bi}$ & $\mathrm{C}$ & $\mathrm{H}$ & $\mathrm{S}$ & \\
\hline $\begin{array}{l}\text { Ethanethiol +1,10- } \\
\text { phenanthroline }\end{array}$ & 40 & 864 & $\begin{array}{c}\mathrm{C}_{6} \mathrm{H}_{15} \mathrm{~S}_{3} \mathrm{Bi} \\
\mathrm{C}_{12} \mathrm{H}_{8} \mathrm{~N}_{2}\end{array}$ & Yellow & $\begin{array}{c}31.2 \\
(35.4)\end{array}$ & $\begin{array}{c}31.2 \\
(36.5)\end{array}$ & $\begin{array}{c}0.6 \\
(4.2)\end{array}$ & $\begin{array}{c}14.1 \\
(16.2)\end{array}$ & 0.91 \\
\hline $\begin{array}{c}\text { 2-propanethiol } \\
+1,10- \\
\text { phenanthroline }\end{array}$ & 60 & 864 & $\begin{array}{c}\mathrm{C}_{9} \mathrm{H}_{24} \mathrm{~S}_{3} \mathrm{Bi} \\
\mathrm{C}_{12} \mathrm{H}_{8} \mathrm{~N}_{2}\end{array}$ & Pink & $\begin{array}{c}30.6 \\
(33.0)\end{array}$ & $\begin{array}{c}32.2 \\
(39.8)\end{array}$ & $\begin{array}{c}4.6 \\
(6.8)\end{array}$ & $\begin{array}{c}14.0 \\
(15.2)\end{array}$ & 0.83 \\
\hline $\begin{array}{c}\text { 2-methylpropane-2- } \\
\text { thiol }+1,10- \\
\text { phenanthroline } \\
\end{array}$ & 50 & 864 & $\begin{array}{c}\mathrm{C}_{12} \mathrm{H}_{27} \mathrm{~S}_{3} \mathrm{Bi} \\
\mathrm{C}_{12} \mathrm{H}_{8} \mathrm{~N}_{2}\end{array}$ & Yellow & $\begin{array}{l}21.8 \\
(30.9)\end{array}$ & $\begin{array}{l}39.6 \\
(42.7)\end{array}$ & $\begin{array}{c}4.7 \\
(5.5)\end{array}$ & $\begin{array}{l}13.7 \\
(14.1\end{array}$ & 0.97 \\
\hline $\begin{array}{c}\text { Butanethiol +1,10- } \\
\text { phenanthroline }\end{array}$ & 40 & 864 & $\begin{array}{c}\mathrm{C}_{12} \mathrm{H}_{27} \mathrm{~S}_{3} \mathrm{Bi} \\
\mathrm{C}_{12} \mathrm{H}_{8} \mathrm{~N}_{2}\end{array}$ & Pink & $\begin{array}{c}29.1 \\
(30.9)\end{array}$ & $\begin{array}{c}40.1 \\
(42.6)\end{array}$ & $\begin{array}{c}4.6 \\
(5.6)\end{array}$ & $\begin{array}{c}12.6 \\
(14.1)\end{array}$ & 0.89 \\
\hline $\begin{array}{c}\text { 1-pentanethiol } \\
+1,10- \\
\text { phenanthroline }\end{array}$ & 40 & 864 & $\begin{array}{c}\mathrm{C}_{15} \mathrm{H}_{33} \mathrm{~S}_{3} \mathrm{Bi} \\
\mathrm{C}_{12} \mathrm{H}_{8} \mathrm{~N}_{2}\end{array}$ & Orange & $\begin{array}{l}26.3 \\
(29.2)\end{array}$ & $\begin{array}{c}40.7 \\
(45.2)\end{array}$ & $\begin{array}{c}4.8 \\
(6.0)\end{array}$ & $\begin{array}{c}11.2 \\
(13.5)\end{array}$ & 0.94 \\
\hline $\begin{array}{c}\text { Ethane-1, 2-dithiol } \\
+1,10- \\
\text { phenanthroline }\end{array}$ & 50 & 864 & $\begin{array}{c}\mathrm{C}_{6} \mathrm{H}_{15} \mathrm{~s}_{6} \mathrm{Bi} \\
\mathrm{C}_{12} \mathrm{H}_{8} \mathrm{~N}_{2}\end{array}$ & Purple & $\begin{array}{l}29.0 \\
(30.4)\end{array}$ & $\begin{array}{c}28.3 \\
(31.4)\end{array}$ & $\begin{array}{c}0.3 \\
(3.6)\end{array}$ & $\begin{array}{l}25.6 \\
(27.9)\end{array}$ & 0.88 \\
\hline $\begin{array}{c}\text { Butane-1, 4-dithiol } \\
+1,10- \\
\text { phenanthroline }\end{array}$ & 50 & 864 & $\begin{array}{c}\mathrm{C}_{12} \mathrm{H}_{27} \mathrm{~S}_{6} \mathrm{Bi} \\
\mathrm{C}_{12} \mathrm{H}_{8} \mathrm{~N}_{2}\end{array}$ & Purple & $\begin{array}{l}26.4 \\
(27.1)\end{array}$ & $\begin{array}{c}29.4 \\
(39.3)\end{array}$ & $\begin{array}{c}3.8 \\
(4.7)\end{array}$ & $\begin{array}{l}23.3 \\
(24.9)\end{array}$ & 0.79 \\
\hline
\end{tabular}

Table-III: Electrolysis Characteristics, Analytical and other Related Data of Electrolytic product of various thiol Systems + 2, 2' - bipyridyl at Bismuth Anode

\begin{tabular}{|c|c|c|c|c|c|c|c|c|c|}
\hline \multirow[t]{2}{*}{ System } & \multirow[t]{2}{*}{$\begin{array}{r}\text { Potent } \\
\text { ial }\end{array}$} & \multirow{2}{*}{$\begin{array}{l}\text { Electricity } \\
\text { Passed } \\
\text { (Coulomb) }\end{array}$} & \multirow[t]{2}{*}{ Product } & \multirow[t]{2}{*}{ Colour } & \multicolumn{4}{|c|}{$\begin{array}{l}\text { Elemental Analysis } \\
\text { Found/(Calc.)\% }\end{array}$} & \multirow{2}{*}{$\begin{array}{l}\text { CurrentEfficienci } \\
\text { es (Gram } \\
\text { Equivalent/ } \\
\text { Faraday) }\end{array}$} \\
\hline & & & & & $\mathrm{Bi}$ & $\mathrm{C}$ & $\mathrm{H}$ & $\mathrm{S}$ & \\
\hline $\begin{array}{l}\text { Ethanethiol }+2,2^{\prime} \\
\text { - bipyridyl }\end{array}$ & 40 & 864 & $\begin{array}{l}\mathrm{C}_{6} \mathrm{H}_{15} \mathrm{~S}_{3} \mathrm{Bi} \\
. \mathrm{C}_{10} \mathrm{H}_{8} \mathrm{~N}_{2}\end{array}$ & $\begin{array}{l}\text { Dirty } \\
\text { Yellow }\end{array}$ & $\begin{array}{c}37.5 \\
(38.1)\end{array}$ & $\begin{array}{c}31.0 \\
(35.3)\end{array}$ & $\begin{array}{c}3.9 \\
(4.4)\end{array}$ & $\begin{array}{c}15.3 \\
(17.5)\end{array}$ & 0.92 \\
\hline $\begin{array}{c}\text { 2-propanethiol+2, } \\
2^{\prime} \text { - bipyridyl }\end{array}$ & 60 & 864 & $\begin{array}{l}\mathrm{C}_{9} \mathrm{H}_{24} \mathrm{~S}_{3} \mathrm{Bi} \\
. \mathrm{C}_{10} \mathrm{H}_{8} \mathrm{~N}_{2}\end{array}$ & Yellow & $\begin{array}{c}36.4 \\
(35.4)\end{array}$ & $\begin{array}{c}30.4 \\
(38.8)\end{array}$ & $\begin{array}{c}2.6 \\
(4.8)\end{array}$ & $\begin{array}{c}14.8 \\
(16.1)\end{array}$ & 0.81 \\
\hline $\begin{array}{c}\text { 2-methylpropane- } \\
\text { 2-thiol }+2,2^{\prime}- \\
\text { bipyridyl }\end{array}$ & 50 & 864 & $\begin{array}{l}\mathrm{C}_{12} \mathrm{H}_{27} \mathrm{~S}_{3} \mathrm{~B} \\
\text { i. } \mathrm{C}_{10} \mathrm{H}_{8} \mathrm{~N}_{2}\end{array}$ & Yellow & $\begin{array}{c}31.5 \\
(33.0)\end{array}$ & $\begin{array}{c}39.7 \\
(41.7)\end{array}$ & $\begin{array}{c}4.0 \\
(5.6)\end{array}$ & $\begin{array}{c}13.7 \\
(15.1)\end{array}$ & 0.87 \\
\hline $\begin{array}{c}\text { Butanethiol+ } 2,2^{\prime} \\
\text { - bipyridyl }\end{array}$ & 40 & 864 & $\begin{array}{l}\mathrm{C}_{12} \mathrm{H}_{27} \mathrm{~S}_{3} \mathrm{~B} \\
\text { i. } \mathrm{C}_{10} \mathrm{H}_{8} \mathrm{~N}_{2}\end{array}$ & Orange & $\begin{array}{c}32.6 \\
(33.0)\end{array}$ & $\begin{array}{l}37.6 \\
(41.7)\end{array}$ & $\begin{array}{c}4.7 \\
(5.6)\end{array}$ & $\begin{array}{c}11.6 \\
(15.1)\end{array}$ & 0.78 \\
\hline $\begin{array}{c}\text { 1-pentanethiol + 2, } \\
\text { 2' - bipyridyl }\end{array}$ & 40 & 864 & $\begin{array}{c}\mathrm{C}_{15} \mathrm{H}_{33} \mathrm{Ns}_{3} \\
\mathrm{Bi} . \\
\mathrm{C}_{10} \mathrm{H}_{8} \mathrm{~N}_{2} \\
\end{array}$ & Orange & $\begin{array}{c}29.8 \\
(31.3)\end{array}$ & $\begin{array}{c}41.3 \\
(44.7)\end{array}$ & $\begin{array}{c}5.5 \\
(6.1)\end{array}$ & $\begin{array}{c}10.2 \\
(14.5)\end{array}$ & 0.91 \\
\hline $\begin{array}{l}\text { Ethane- 1, 2- } \\
\text { dithiol+ 2, 2' - }\end{array}$ & 50 & 864 & $\begin{array}{l}\mathrm{C}_{6} \mathrm{H}_{15} \mathrm{~S}_{6} \mathrm{Bi} \\
. \mathrm{C}_{10} \mathrm{H}_{8} \mathrm{~N}_{2}\end{array}$ & Purple & $\begin{array}{c}30.6 \\
(32.8)\end{array}$ & $\begin{array}{c}26.3 \\
(29.7) \\
\end{array}$ & $\begin{array}{c}2.8 \\
(3.5)\end{array}$ & $\begin{array}{c}27.6 \\
(29.3)\end{array}$ & 0.81 \\
\hline
\end{tabular}


Direct electrochemical synthesis of Bismuth (III) thiolates/dithiolates and their coordination ..

\begin{tabular}{|c|c|c|c|c|c|c|c|c|c|}
\hline bipyridyl & & & & & & & & & \\
\hline $\begin{array}{c}\text { Butane-1, 4- } \\
\text { dithiol+ 2, 2' } \\
\text { bipyridyl }\end{array}$ & 40 & 864 & $\mathrm{C}_{12} \mathrm{H}_{27} \mathrm{~S}_{6} \mathrm{~B}$ & Purple & 26.6 & 15.6 & 0.6 & 21.3 & 0.84 \\
i. $\mathrm{C}_{10} \mathrm{H}_{8} \mathrm{~N}_{2}$ & & $(28.5)$ & $(36.1)$ & $(4.8)$ & $(23.5)$ & \\
\hline
\end{tabular}

Current efficiencies of some of these systems have also determined which are quite close to $100 \%$, thereby indicating that the reactions leading to formation of coordination compounds are the predominant reactions of these systems.

\section{Conclusion}

The project studies thus reveals that electrochemical method for synthesis of bismuth thiolates and their coordination compounds is a single step process and is quite simple as compared to other reported multistep methods for the synthesis of these compounds.

\section{References}

[1] Baljit Singh and Harpreet Kaur, Direct electrochemical synthesis of bismuth (III) phenoxides and their coordination compounds, EJournal of Chemistry, 9(1), 2012, 381-388.

[2] J.S. Banait, Baljit Singh and Harpreet Kaur, J. Indian. Chem. Soc, 56, 2008, 156.

[3] Baljit Singh and Harpreet Kaur, Electrochemical reactions at sacrificial electrodes. Part-XXIII: Direct electrochemical synthesis of bismuth (III) alkoxides, J. Indian. Chem. Soc, 85, 2008, 849-851.

[4] J. Mihilcic, K.Moeller, J. Am. Chem. Soc, 125, 2003, 36-37.

[5] J. Mihilcic, K.Moeller, J. Am. Chem. Soc, 126, 2004, 9106-9111.

[6] J. Tian, K. D. Moeller, Org. Lett, 7, 2005, 5381-5383.

[7] I. Kappstein, I. Engles, Eur. J. Clin. Micro, 6,1987, 216.

[8] A. Naganuma, M. Satoh, N. Imura, Cancer Research, 47, 1987, 983.

[9] P. J. Sadler, H. Li, H. Sun, Coordination Chemistry Reviews, 185-186, 1999, 689-709.

[10] G. G. Briand, N. Burford, Chemical Reviews, 99, 1999, 2601-2657.

[11] A.I Vogel, A Text Book of Quantitative Inorganic Analysis (Longman Green, London, 1961).

[12] Otsuka, S, Kamata, M., J. Am. Chem. Soc. 103, 1981, 3011-3014.

[13] P. Boudjouk, M.P Remington, Jr., D. G. Griaer, B. R. Jarabek and G. J. McCarthy, Inorg. Chem, 37, 1998, 3538-3541

[14] G. G. Briand, N. Burford, T. S. Cameron and W. Kwiatkowski, J. Am. Chem. Soc,120, 1998, 11374-11379.

[15] L. Agocs, G. G. Briand, N. Burford, T. S. Cameron and W. Kwiatkowski and K.N. Robertson, Inorg. Chem, 36, 1997, $2855-2860$.

[16] H. Ylin, F. Li and D.Wang, J. Coord. Chem, 60(11), 2007, 1133-1141.

[17] J.R. Dyer, Application Of Absorption Spectroscopy Of Organic compounds, (Prentice Hall Of India Pvt. Ltd., New Delhi, 1978.)

[18] M. A. Kirsty, J. B. Christian, C.N. Nicholas, O. A. Guy, J. Starbuck, Dalton Transactions, 16, 2003, 3270-3277. 